\title{
ANÁLISE DA PRECIPITAÇÃO SAZONAL SIMULADA ULTILIZANDO 0 REGCM4 SOBRE O ESTADO DO PARÁ EM ANOS DE EXTREMOS CLIMATICOS
}

\author{
ÁVILA, Pâmela Lorena Ribeiro - panloly@hotmail.com \\ Mestranda em Meteorologia, Universidade Federal de Campina Grande \\ SOUZA, Everaldo Barreiros de - everaldo@ufpa.br \\ Doutor, Universidade Federal do Pará \\ PINHEIRO, Amanda Nascimento - nanda_tkr18@hotmail.com \\ Mestranda em Ciências ambientais, Universidade Federal do Pará \\ FIGUEIRA, Waléria Souza - wal.figueira@gmail.com \\ Mestranda em Meteorologia, Universidade Federal de Campina Grande
}

\begin{abstract}
RESUMO: Este trabalho apresenta uma contribuição aos estudos de modelagem climática com ênfase na variabilidade pluviométrica sazonal do estado do Pará, durante as estações de verão e outono (DJF e MAM). Baseado nos resultados das simulações regionais do RegCM4 para os anos de El Niño (1982/83) e Lã Niña (1988/89) usando domínio em alta resolução espacial $(50 \mathrm{Km}$ ) e o esquema de convecção MIT, foi investigado o desempenho do modelo em simular a distribuição regional de precipitação sazonal no estado do Pará. As análises quantitativas evidenciaram que o RegCM4 apresenta mais representativo na grade G2 para o ano de El Niño. Além disso, através da técnica de composições, também foi investigado o desempenho do RegCM4 em reproduzir os padrões espaciais anômalos de precipitação sazonal em associação aos episódios ENOS, e as fases do gradiente térmico sobre o Atlântico intertropical. Os resultados demonstraram que o modelo conseguiu representar realisticamente bem 0 padrão espacial das anomalias pluviométricas acima (abaixo) do normal em grande parte da Amazônia oriental, durante os conhecidos cenários favoráveis, i.e., condições de La Niña e gradiente de aTSM para o Atlântico sul (desfavoráveis, i.e., El Niño e gradiente de aTSM para o Atlântico norte).
\end{abstract}

Palavras-chave: Precipitação sazonal, El Niño, Lã Niña. ENOS. RegCM4

SIMULATION OF SEASONAL PRECIPITATION WITH REGCM4 ABOUT THE STATE OF PARÁ FOR YEARS OF EL NIÑO AND LA NIÑA

\begin{abstract}
This work presents a contribution to climate modeling studies with an emphasis on seasonal rainfall variability in the state of Pará, during the seasons of summer and autumn ( DJF and MAM ). Based on the results of the regional simulations RegCM4 for El Niño years ( $1982 / 83$ ) and La Niña ( $1988 / 89$ ) using the field at high spatial resolution ( $50 \mathrm{Km}$ ) and the MIT convection scheme, we investigate the performance of the model in simulate the regional distribution of seasonal rainfall in the state of Pará quantitative analysis showed that the most representative features RegCM4 the G2 grid to the El Niño year. Moreover, using the technique of composition, we also investigated the performance of RegCM4 to reproduce the anomalous spatial patterns of seasonal rainfall in association with ENSO episodes, and the phases of the thermal gradient on the intertropical Atlantic. The results showed that the model was able to realistically represent well the spatial pattern of rainfall anomalies above (below ) normal in much of the eastern Amazon, known for favorable scenarios, ie, La Niña conditions and gradient SSTa south Atlantic ( unfavorable, ie, El Niño and gradient SSTa north Atlantic ).
\end{abstract}

Key words: seasonal rainfall, El Nino, La Nina, Amazon, ENSO, RegCM4 


\section{INTRODUÇÃO}

A característica intrínseca do clima da Amazônia é a presença de um amplo espectro de variações no tempo e espaço da atividade convectiva tropical e da precipitação, as quais se tornam as variáveis climáticas mais importantes da região.

No contexto da dinâmica climática tropical, é bem conhecido que a variabilidade interanual e sazonal da estação chuvosa da Amazônia é modulada diretamente pelos padrões oceano-atmosfera de grande escala, associados ao ciclo do El Niño-Oscilação Sul (ENOS) sobre o Oceano Pacífico e as fases do gradiente meridional inter hemisférico de anomalias de temperatura da superfície do mar (aTSM) sobre o Oceano Atlântico intertropical (NOBRE; SHUKLA, 1996; SOUZA et al., 2000).

Através de mudanças significativas nos padrões da circulação troposférica associados às células de Walker e de Hadley (SOUZA; AMBRIZZI, 2002; SOUZA; AMBRIZZI; KAYANO, 2004), ambos os modos climáticos do Pacífico e Atlântico interferem na posição e intensidade das bandas de nebulosidade convectiva da ZCAS e ZCIT e, portanto, modulam a distribuição de chuva na região.

As análises observacionais conduzidas por Souza, Ambrizzi e Kayano. (2005), considerando dois cenários climáticos contrastantes denominados de favorável: concomitância de condições de La Niña e gradiente meridional de aTSM apontando para a bacia do Atlântico sul (desfavorável: concomitância de condições de El Niño e gradiente meridional de aTSM apontando para a bacia do Atlântico norte), evidenciaram a predominância de circulação atmosférica ascendente (descendente) nas regiões da célula de Walker/Hadley equatorial, que fortaleceu (inibiu) a ZCIT e consequentemente resultou em precipitação sazonal significativamente abundante (deficitária) no leste da Amazônia.

A maior parte desta precipitação anual ocorre entre as estações de verão e outono austral (MARENGO et al., 2001; SOUZA; AMBRIZZI, 2003), tipicamente de dezembro a maio, em associação aos padrões de circulação atmosférica quase-estacionários de grande escala associados à Zona de Convergência do Atlântico Sul (ZCAS) e Zona de Convergência Intertropical (ZCIT) (SOUZA, AMBRIZZI; KAYANO, 2004, SOUZA; ROCHA, 2006).

A ZCAS é um fenômeno meteorológico de escala intrasazonal que influencia a distribuição de precipitação, sendo em muitos casos, responsável por variabilidades no clima regional (KODAMA, 1992; LIEBMANN et al., 1999; NOGUES-PAEGLE; MO, 1997). Assim sendo, o Pará também está sujeito aos efeitos da ZCAS, que tem sua maior freqüência nos meses de verão, coincidindo com a estação chuvosa e afetando os índices pluviométricos, principalmente na porção Sul do estado. É comum nestes episódios, haver um acoplamento da ZCAS com a ZCIT, em associação com sistemas como a Alta da Bolívia e vórtices ciclônicos em altos níveis, o que pode provocar chuvas generalizadas sobre grande parte do estado (GAN; SOUZA; AMBRIZZI, 2003). 
A ZCIT, por sua vez, exerce um papel preponderante na modulação do regime pluviométrico na região equatorial, podendo ser considerada o sistema meteorológico de grande escala de maior importância no Pará, visto que concorre para o estabelecimento do período chuvoso do estado no decorrer de sua marcha em direção ao Hemisfério Sul, durante o verão e outono austral. Através de sua climatologia, Waliser e Gautier (1993) mostraram a importância deste sistema na definição e análise do clima terrestre em escala global e local. Assim, a sua variabilidade sazonal e interanual em decorrência de interações com as condições oceânicas, principalmente no Atlântico Tropical, têm implicação direta na duração e intensidade da estação chuvosa paraense. Por outro lado, a ocorrência de sistemas de mesoescala, como as linhas de instabilidade, pode ser responsável por até $45 \%$ da precipitação na parte Leste do Pará (COHEN; DIAS; NOBRE, 1989).

Diversos trabalhos têm mostrado ao longo dos últimos anos a relevância da modelagem numérica para simular o clima presente e, principalmente, o futuro. Entretanto, modelos de circulação geral (MCG) não são capazes de resolver satisfatoriamente os detalhes da fisiografia, assim como aqueles da circulação local. Todavia, visto que os MCG apresentam resolução espacial em torno de uma centena de quilômetros ou até mais, aqueles não são plenamente capazes de perceber forçantes de pequena ou mesoescala, da mesma forma como determinados aspectos da cobertura do solo, a presença de corpos d'água e, ainda, processos físicos dinâmicos de subgrade, dentre eles radiação, convecção, processos turbulentos, etc. (SETH et al., 2007). Estas limitações certamente prejudicam o prognóstico de variáveis meteorológicas em escala regional, especialmente a precipitação.

Naturalmente que o aumento da resolução espacial de um MCG deveria conduzir a um melhor prognóstico, todavia, o custo computacional para realizar tal processamento, além de um aumento exagerado na capacidade de armazenamento exige outras alternativas. Não obstante, o ajuste adequado das parametrizações convectivas em escala global não seria tarefa das mais triviais, podendo levar a resultados extremamente equivocados em determinadas regiões (ALVES, 2007).

Nesse sentido, os modelos climáticos globais e regionais são reconhecidamente ferramentas chave, uma vez que os mesmos buscam equacionar o sistema climático e assim investigar com maior detalhamento os mecanismos ou processos reguladores do clima e sua variabilidade espaço-temporal.

No contexto dos estudos de modelagem regional, há praticamente um consenso de que os processos ou mecanismos dinâmicos são relativamente bem representados nas simulações. Porém, a grande maioria desses estudos abordou análises sazonais e resolução espacial variando de 50 a $80 \mathrm{~km}$, sendo que as validações de precipitação sazonal são enfatizadas para a região Sudeste e Nordeste do Brasil, ficando a região da Amazônia em segundo plano. Até o presente, portanto, os resultados obtidos na modelagem abordaram aspectos da chuva numa escala de baixa resolução e considerando análises sazonais. Poucos estudos na escala mensal foram realizados, sobretudo para a região Amazônica, tampouco para o estado paraense. 


\subsection{OBJETIVOS}

O objetivo geral é investigar a escolha do domínio geográfico (tamanho da grade) na simulação da precipitação sazonal (verão e outono austral) da Amazônia oriental, durante anos de contraste climático (ano chuvoso e seco). Objetivos específicos:

1. Analisar os padrões (distribuição espacial e intensidade) da precipitação sazonal observada sobre a Amazônia oriental, durante o verão/outono de 1982/83 (ano seco) e 1988/89 (ano chuvoso);

2. Comparar e analisar os campos de precipitação sazonal observada e simulada sobre a Amazônia oriental para as diferentes grades do modelo climático regional durante os anos de contraste climático, caracterizados como anomalamente chuvoso e seco.

\section{MATERIAIS E MÉTODOS}

\subsection{DADOS}

Os dados de precipitação sobre a Amazônia são provenientes do conjunto de dados pertencente ao Centro de Previsão Climática (CPC) do National Centers for Environmental Prediction (NCEP) dos EUA. Estes dados encontram-se documentados em Silva et al. (2007) e foram gerados através da interpolação espacial dos dados observados nas estações meteorológicas de superfície, reportados na codificação SYNOP das 1200UTC. Maiores detalhes sobre o controle de qualidade e métodos e procedimentos metodológicos podem ser encontrados em Silva et al. (2007).

Utilizam-se também os dados de TSM semanal (REYNOL et al.DS , 2002) e as variáveis meteorológicas: componentes zonal e meridional do vento, velocidade vertical, temperatura do ar, altura geopotencial, umidade relativa e pressão ao nível médio do mar do conjunto de reanálise NCEP/NCAR (KALNAY et al., 1996).

\subsection{MODELO CLIMÁTICO REGIONAL REGCM4}

O RegCM4 usa o esquema de superfície Biosphere-Atmosphere Transfer Scheme (DICKINSON; HENDERSON-SELLERS; KENNEDY, 1993) para resolver, em cada ponto da grade, os processos de interação da superfície (tipos de vegetação e solo) com a atmosfera, levando-se em consideração os processos de trocas turbulentas de momentum, energia e vapor de água. O transporte turbulento dessas grandezas resulta do produto entre o respectivo gradiente vertical e o coeficiente de difusão vertical turbulenta segundo as correções propostas por Holtslag. Para a transferência radiativa utiliza-se o esquema do Community Climate Model 3 (KIEHL et al., 1996), no qual as taxas de aquecimento e fluxos na superfície para a radiação solar e infravermelho sob condições de céu claro e nublado são calculadas separadamente.

A parametrização MIT-Emanuel, que é uma parametrização de convecção de cumulus recente para o modelo RegCM4. Este esquema considera que a mistura nas nuvens é altamente episódica e não homogênea, cujos fluxos convectivos são baseados em um modelo de correntes ascendentes e descendentes de escalas de sub-nuvem (EMANUEL, 1991). A convecção é, então, iniciada quando 
o nível de empuxo neutro é maior que o nível de condensação por levantamento na base da nuvem. Entre estes dois níveis, o ar se eleva e uma parte da fração de umidade condensada forma precipitação, enquanto a outra parte restante forma nuvem (EMANUEL; ZIVKOVIC-ROTHMAN, 1999). A nuvem, por sua vez, mistura-se com o ar ambiente de acordo com um espectro uniforme de misturas que conduz a parcela para cima ou para baixo, atingindo, assim, o nível de empuxo neutro. As taxas de entranhamento e desentranhamento são calculadas através dos gradientes verticais de flutuabilidade na nuvem.

\subsection{CONFIGURAÇÃO DAS RODADAS DO MODELO}

As simulações foram realizadas utilizando como condições de contorno inferior e lateral (ICBC) os campos de TSM semanal do NCEP e as variáveis meteorológicas da reanálise NCEP/NCAR, respectivamente. As rodadas iniciaram no dia 15 de Novembro e finalizaram em 31 de Maio do ano seguinte, ou seja, o tempo de integração é de 7 meses, sendo que o primeiro mês (novembro) é considerado o spin-up do modelo e é descartado. Todas as simulações foram geradas com resolução horizontal de $50 \mathrm{Km}$, centrado em $2^{\circ} \mathrm{S} / 49^{\circ} \mathrm{W}$ com 18 níveis na vertical (sendo 7 níveis abaixo de $800 \mathrm{hPa}$ ) e usando projeção mercator normal.

Para se investigar a escolha do domínio nas simulações do modelo RegCM4 adotou-se 3 grades com dimensionamento diferenciado, conforme mostra a Figura 1.

A grade 1 (G1) com 48 x 48 pontos nas direções x (longitude) e y (latitude) que engloba a Amazônia oriental e uma pequena porção do Oceano Atlântico equatorial;

A grade 2 (G2) com 96 x 96 pontos nas direções x (longitude) e y (latitude) que engloba grande parte da América do Sul e Oceano Atlântico tropical;

A grade 3 (G3) com 192 x 104 pontos nas direções x (longitude) e y (latitude) que engloba os Oceano Pacífico e Atlântico tropical e a América do Sul e África.

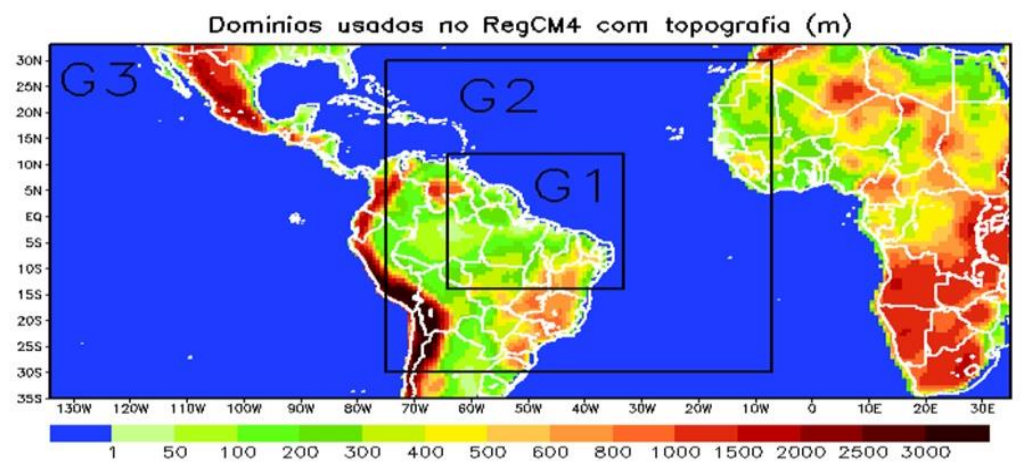

Figura 1. Domínios geográficos usados nas simulações do modelo regional. G1, G2 e G3 representam as grades do Pacífico+América do Sul+Atlântico+África, América do Sul+Atlântico, e Amazônia, respectivamente. As cores representam a topografia (m). 


\section{RESULTADOS}

\subsection{CONDIÇÕES CLIMÁTICAS DE GRANDE ESCALA}

Nesta secção apresentam-se os dois anos de contraste climático caracterizados por mecanismos de grande escala inversos, conforme descrições detalhadas a seguir.

\subsubsection{Condições climáticas observadas em 1982/83:}

A Figura 2 mostra as anomalias de TSM observada sobre as bacias dos Oceanos Pacífico e Atlântico e os padrões da circulação atmosférica zonal associada à célula de Walker, durante os períodos sazonais do verão (DJF 1982/83) e outono (MAM, 1983). Nota-se claramente a presença de anomalias positivas de TSM (indicando a ocorrência de águas oceânicas mais quentes do que o normal) cobrindo a maior parte do Pacífico tropical (Figura 2, mapas inferiores). Essa configuração denota a ocorrência do episódio El Niño de intensidade forte (anomalias alcançam magnitudes acima de $2^{\circ} \mathrm{C}$ ) atuando em DJF e MAM de 1982/83. Na bacia do Oceano Atlântico, verifica-se anomalias negativas no setor equatorial e na parte norte da bacia durante DJF. Já em MAM, configura-se um padrão invertido de anomalias positivas na bacia norte e anomalias negativas na bacia sul do Oceano Atlântico. Essa configuração indica a atuação da fase do dipolo positivo - ou gradiente térmico, apontando para a bacia norte do Atlântico (Figura 2). Portanto, os períodos do verão e outono de 1982/83 foram marcados pela manifestação do El Niño no Pacífico e do Dipolo positivo no Atlântico, os quais são mecanismos desfavoráveis à ocorrência de chuvas na Amazônia.

Na Figura 2 nota-se ainda o ramo ascendente (vetores com direção para cima) da célula de Walker ocorrendo sobre o Pacífico, coincidente com a região de aquecimento anômalo do El Niño, durante DJF e MAM. Um ramo descendente (vetores com direção para baixo) compensatório é observado sobre o leste da Amazônia e Atlântico equatorial, principalmente em DJF (Figura 2, mapas superiores). Enquanto que, em MAM, devido a possível atuação do dipolo positivo, este enfraquece os ventos descendentes nessa região.

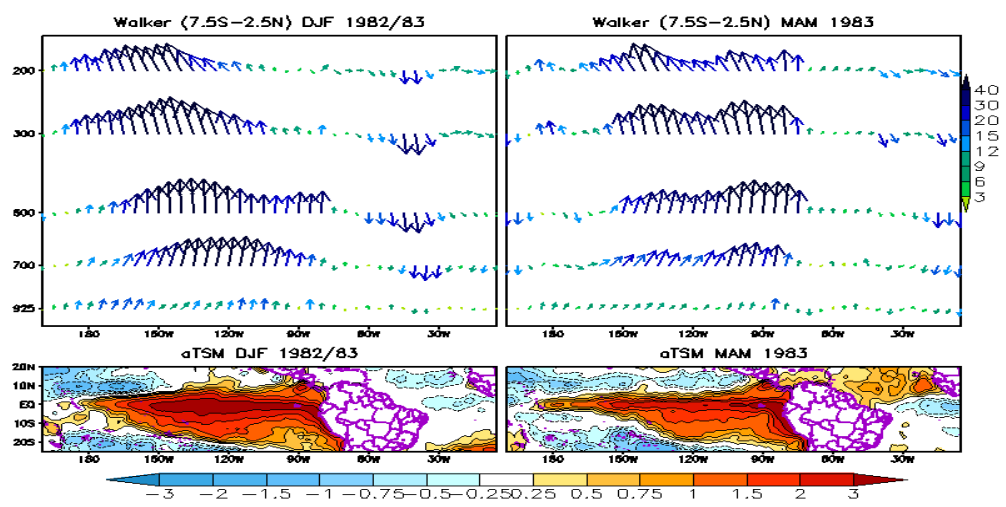

Figura 2. Cortes verticais (longitude $x$ altura) da circulação zonal associada à célula de Walker média entre $7.5^{\circ} \mathrm{S}-2.5^{\circ} \mathrm{N}$ (mapa superior) e anomalias de TSM (mapa inferior) sobre o Pacífico e Atlântico durante DJF 1982/83 e MAM 1983. 


\section{ISSN: 1980-055x (Impressa) 2237-8642 (Eletrônica)}

A Figura 3 mostra as anomalias de ROL e os padrões da circulação atmosférica meridional associada à célula de Hadley, durante os períodos sazonais do verão (DJF 1982/83) e outono (MAM 1983). Observa-se um forte ramo de ar descendente centrado na região equatorial, atingindo a Amazônia oriental, durante o período de DJF e verifica-se uma intensa anomalia de ROL (com valores alcançando a magnitude de até $30 \mathrm{~W} / \mathrm{m}^{2}$ ) na porção norte do Pará. Já para MAM verifica-se uma circulação mais enfraquecida na região equatorial do que na figura anterior, provavelmente por conta do aparecimento de um ROL negativo entre $5^{\circ} \mathrm{N}$ e $10^{\circ} \mathrm{N}$ e outra área com valores mais baixos de ROL $\left(5^{\circ} \mathrm{S}\right.$ e $10^{\circ} \mathrm{S}$ ), o que favorece moderado vento ascendente nestas áreas e predomínio do movimento descendente com vetores de pequena magnitude sobre o Equador. Conseqüentemente, essa área em estudo se torna desfavorável à ocorrência de convecção para ambos os períodos, que teria um maior pico em anos normais.

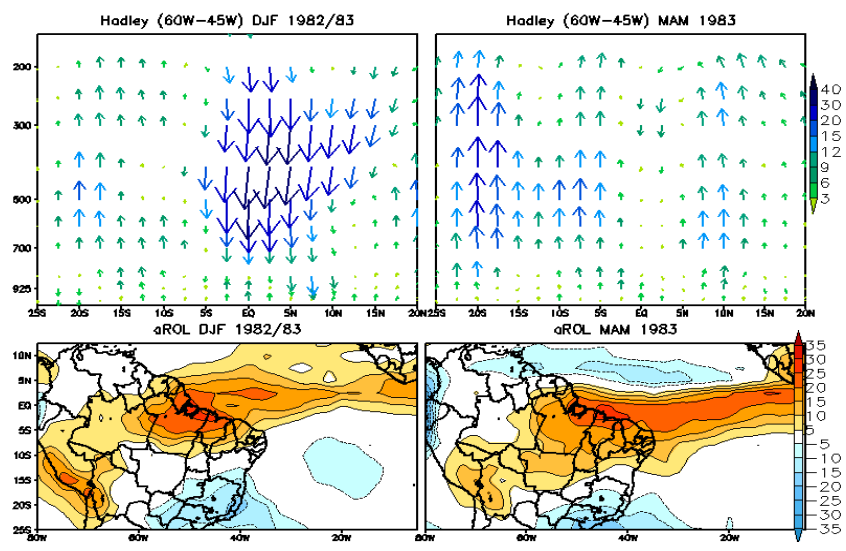

Figura 3. Cortes verticais (latitude $x$ altura) da circulação meridional associada à célula de Hadley média entre 600 $-45^{\circ} \mathrm{W}$ (mapa superior) e anomalias de ROL (mapa inferior) sobre a América do Sul e Atlântico durante DJF 1982/83 e MAM 1983.

A Figura 4 mostra os padrões de vento horizontal em $200 \mathrm{hPa}$ (mapa superior) e em $850 \mathrm{hPa}$ (mapa inferior) durante DJF 1982/83 e MAM 1983 observado. Os efeitos dos padrões anômalos já comentados acima vão influenciar consideravelmente na circulação tanto em altos níveis (200 hPa) como em baixos níveis ( $850 \mathrm{hPa}$ ), mostrando que para o período de $\mathrm{DJF}$ em altos níveis não se tem a presença da Alta da Bolívia, sendo que nota-se apenas a presença de uma crista seguida de um cavado sobre o Pará e em baixos níveis vamos ter os ventos com vetores intensos sobre o oceano e continente vindo de leste e mudando para o sentido noroeste-sudeste para o sul do Brasil. Para o trimestre de MAM verifica-se a presença da Alta da Bolívia enfraquecida com sua área de atuação em altos níveis sobre a Amazônia ocidental e em baixos níveis um padrão semelhante ao encontrado no trimestre de DJF com a diferença de que o vento vai estar posicionado no sentido norte-sul. 

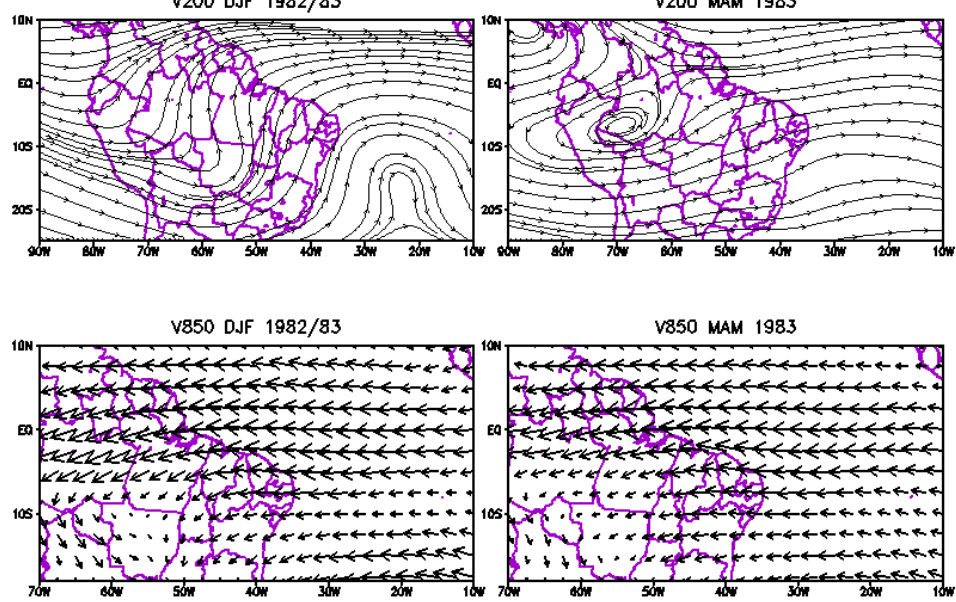

Figura 4. Padrões de vento horizontal em $200 \mathrm{hPa}$ (mapa superior) e em $850 \mathrm{hPa}$ (mapa inferior) durante DJF 1982/83 e MAM 1983.

A Figura 5 mostra as anomalias de precipitação do CPC observada durante DJF 1982/83 e MAM 1983. Observa-se o predomínio de anomalia negativa de precipitação para o trimestre de DJF sobre a maior parte do Pará, apresentando as maiores anomalias no oeste e parte do norte e nordeste com índices de -4 e $3 \mathrm{~mm} / \mathrm{mês}$ respectivamente e mantendo o sul, sudeste e sudoeste com anomalia neutra. Enquanto para o trimestre de MAM esta anomalia se desentensifica diminuindo sua área de atuação no oeste, parte do noroeste e norte indo de uma anomalia de -4 e -3 para $-2 \mathrm{~mm} / \mathrm{mês}$ na maior parte, e intensificando-se na parte nordeste e aumentando a área de anomalia na parte sudeste e sudoeste do Pará, com picos de até de $-5 \mathrm{~mm} / \mathrm{mês}$ no extremo nordeste.

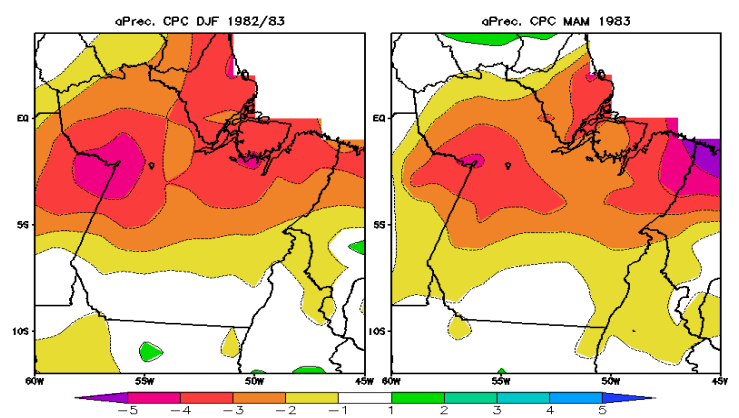

Figura 5. Anomalias de precipitação em $\mathrm{mm} / \mathrm{mês}$ do CPC observada durante DJF 1982/83 e MAM 1983.

\subsubsection{Condições climáticas observadas em 1988/89:}

A Figura 6 mostra as anomalias de TSM observada sobre as bacias dos Oceanos Pacífico e Atlântico e os padrões da circulação atmosférica zonal associada à célula de Walker, durante os períodos sazonais do verão (DJF 1988/89) e outono (MAM 1989). Nota-se claramente a presença de anomalias negativas de TSM 


\section{ISSN: 1980-055x (Impressa) 2237-8642 (Eletrônica)}

(indicando a ocorrência de águas oceânicas mais frias do que o normal) cobrindo a maior parte do Pacífico tropical (Figura 6, mapas inferiores). Essa configuração denota a ocorrência do episódio Lã Niña de intensidade forte (anomalias alcançam magnitudes acima de $-2^{\circ} \mathrm{C}$ ) atuando em DJF e MAM de 1988/89. Na bacia do Oceano Atlântico, verifica-se anomalias negativas no setor equatorial e na parte norte da bacia durante DJF. Já em MAM, configura-se um padrão invertido de anomalias negativas na bacia norte e anomalias positivas na bacia sul do Oceano Atlântico. Essa configuração indica a atuação da fase do dipolo negativo ou gradiente térmico, apontando para a bacia sul do Atlântico (Figura 6). Portanto, os períodos do verão e outono de $1988 / 89$ foram marcados pela manifestação do La Niña no Pacífico e do Dipolo negativo no Atlântico, os quais são mecanismos favoráveis à ocorrência de chuvas na Amazônia.

Na Figura 6 nota-se o ramo descendente (vetores com direção para baixo) da célula de Walker ocorrendo sobre o Pacífico, coincidente com a região de resfriamento anômalo da La Niña, durante DJF e MAM. Além disso, um ramo ascendente (vetores com direção para cima) compensatório é observado sobre o leste da Amazônia e Atlântico equatorial, principalmente em DJF (Figura 6, mapas superiores). Opostamente ao ano de El niño, observa-se o favorecimento da magnitude dos vetores do vento no período de DJF e um pequeno enfraquecimento em MAM, devido a possível atuação do dipolo negativo no norte do Atlântico.

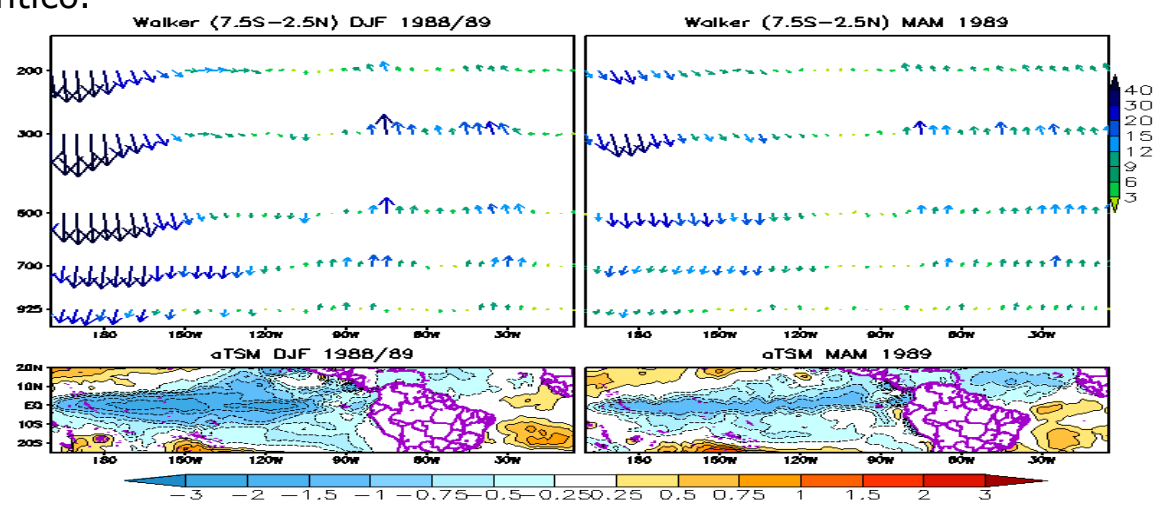

Figura 6. Cortes verticais (longitude $x$ altura) da circulação zonal associada à célula de Walker média entre 7.50 $-2.5^{\circ} \mathrm{N}$ (mapa superior) e anomalias de TSM (mapa inferior) sobre o Pacífico e Atlântico durante DJF 1982/83 e MAM 1983.

A Figura 7 mostra as anomalias de ROL e os padrões da circulação atmosférica meridional associada à célula de Hadley, durante os períodos sazonais do verão (DJF 1988/89) e outono (MAM 1989). Observa-se um ramo de ar ascendente centrado na região equatorial, atingindo a Amazônia oriental, durante o período de DJF e verifica-se uma anomalia negativa de ROL moderada na parte norte e noroeste do Pará (com valores alcançando a magnitude de até $-15 \mathrm{~W} / \mathrm{m}^{2}$ ) e as demais áreas do estado em condições de neutralidade em relação a esta variável. Para o período de MAM temos o domínio do ar ascendente com intensidade moderada que atinge a Amazônia oriental, já quando analisamos a anomalia de ROL verifica-se que a área de atuação de anomalia negativa se expandiu sobre quase todo o estado e mantendo sua intensidade moderada com algumas áreas um pouco mais intensa (com valores de magnitude oscilando 
entre -15 e $-20 \mathrm{~W} / \mathrm{m}^{2}$ ) , o que indica que a área em estudo está favorável a ocorrência de chuva.

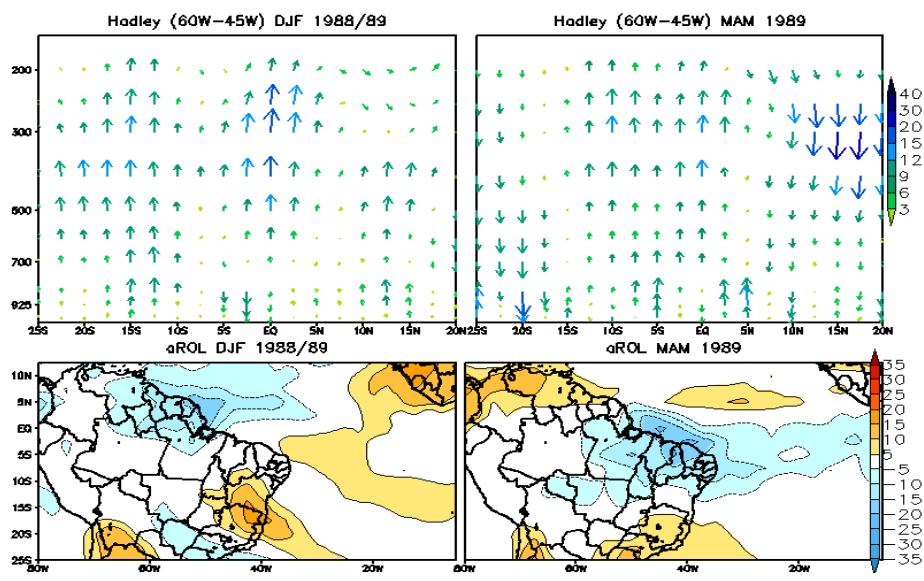

Figura 7. Cortes verticais (latitude $x$ altura) da circulação meridional associada à célula de Hadley média entre $60^{\circ} \mathrm{W}-45^{\circ} \mathrm{W}$ (mapa superior) e anomalias de ROL (mapa inferior) sobre a América do Sul e Atlântico durante DJF 1988/89 e MAM 1989.

A Figura 8 mostra os padrões de vento horizontal em $200 \mathrm{hPa}$ (mapa superior) e em $850 \mathrm{hPa}$ (mapa inferior) durante DJF 1988/89 e MAM 1989 observado. Verifica-se em ambos os trimestres, em altos níveis, a atuação da Alta da Bolívia, sendo esta centrada sobre a Bolívia com seu cavado atuando sobre o Pará e NEB em DJF, e para MAM, é deslocado para o centro-oeste do Brasil com parte do seu cavado atuando no extremo norte do Brasil e oceano Atlântico. Em baixos níveis, similarmente ao ano de El Niño, observa-se os ventos com vetores intensos sobre o oceano e continente vindo de leste e mudando para o sentido noroeste-sudeste para o sul do Brasil para DJF. Enquanto que, para o trimestre de MAM houve um enfraquecimento dos ventos no sentido norte-sul. 

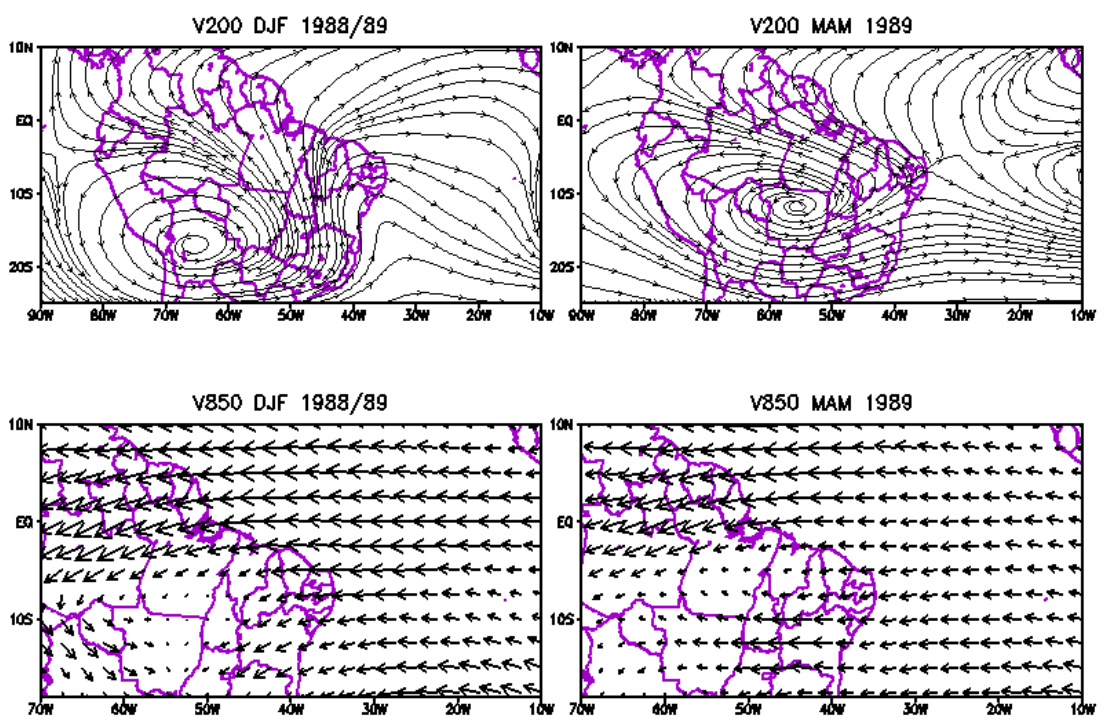

Figura 8. Padrões de vento horizontal em $200 \mathrm{hPa}$ (mapa superior) e em 850 hPa (mapa inferior) durante DJF 1988/89 e MAM 1989.

A Figura 9 mostra anomalias de precipitação do CPC observada durante DJF $1988 / 88$ e MAM 1989. Observa-se que o trimestre de DJF apresenta valores de anomalias de +1 na faixa que vai de parte no nordeste até o sudoeste apresentando alguns pontos de anomalia igual $a+2$ e as demais áreas neutras com pontos de anomalia negativa entre o sudeste e este. Enquanto no trimestre de MAM se apresenta uma grande área influenciada por anomalias acima da media com exceção de áreas no noroeste e sudeste que estão neutras. Os picos estão em parte do oeste, norte e este com anomalias entre +3 e $+4 \mathrm{~mm} / \mathrm{mês}$.

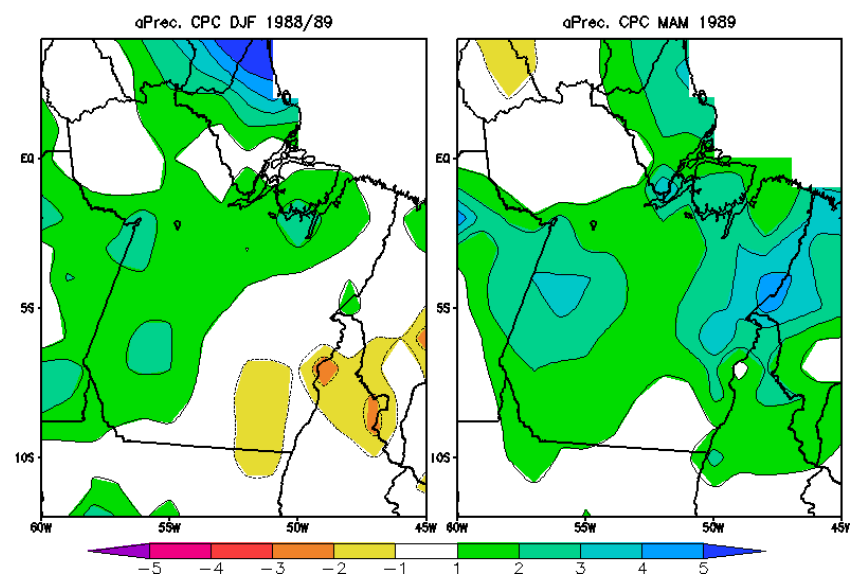

Figura 9. Anomalias de precipitação em $\mathrm{mm} / \mathrm{mês}$ do CPC observada durante DJF 1988/89 e MAM 1989.

\subsection{COMPARAÇÕES DA PRECIPITAÇÃO OBSERVADA E SIMULADA}


A Figura 10 mostra os padrões (distribuição espacial e intensidade) da precipitação sazonal observados nas estações de verão (DJF 1982/83 e 1988/89) e outono (MAM de 1983 e 1989), bem como os padrões climatológicos dos últimos 30 anos (média 1979-2008). Observa-se que em DJF 1982/83 a distribuição da chuva no Pará se manteve inferior ao observado pela climatologia para este período, evidenciando valores mínimos e mais expressivos no noroeste, norte e nordeste do estado. Enquanto em MAM 1983 nota-se que este déficit persistiu e se mostrou expressivo para o noroeste e sudeste do estado. A explicação para que esses valores de precipitação a baixo da média para os dois períodos em estudo tenham ocorrido pode ser observado na Figura 2, onde mostra claramente a presença de anomalias positivas de TSM sobre o Pacifico equatorial caracterizando o fenômeno conhecido como El Niño, que se intensificou no outono (MAM) e também devido ao padrão de dipolo positivo que se estabeleceu no Oceano Atlântico.

Observa-se que em DJF 1988/89 a chuva apresentou valores acima da media climatológica, mostrando-se mais expressiva nas regiões norte, nordeste e sudoeste do estado. Enquanto em MAM de 1989 verifica-se uma intensificação desse padrão em todo o estado. O motivo para estes valores acima da média para esses dois períodos deve-se a presença de anomalias negativas de TSM sobre o pacifico equatorial, caracterizando a La Niña, que se intensificou no outono (MAM) e também devido ao padrão de dipolo negativo que se estabeleceu no Oceano Atlântico favorecendo a precipitação no Pará.

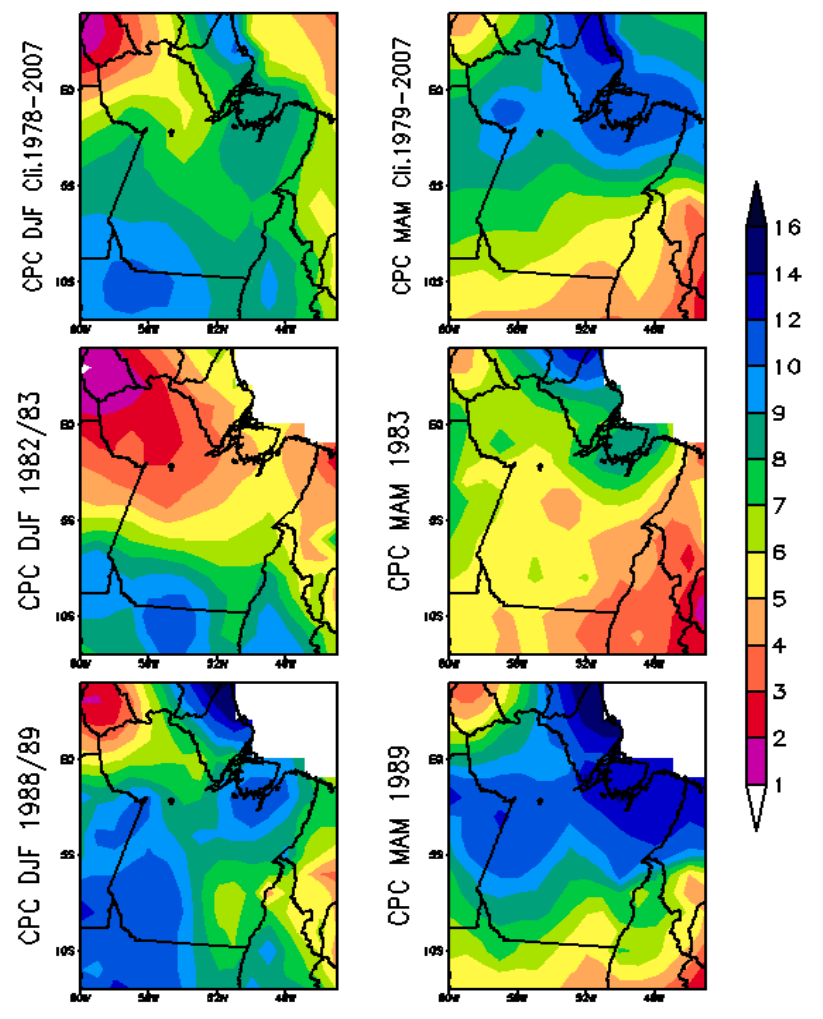

Figura 10. Padrões (distribuição espacial e intensidade) da precipitação ( $\mathrm{mm} / \mathrm{dia}$ ) sazonal observada em DJF e MAM correspondentes a climatologia 1979-2008 (mapa superior), 1982/83 (mapa intermediário) e 1988/89 (mapa inferior). 
A Figura 12 mostra os padrões de precipitação simulados pelo modelo RegCM4 para DJF 1982/83 e MAM 1983 considerando as grades G1, G2 e G3, usando o esquema de parametrização convectiva MIT. Fazendo-se a comparação dos mapas de chuva da Figura 12 (simulados) com os mapas da Figura 10 (observados), observa-se que para DJF a grade G1 do modelo mostra-se representativa para pequenas áreas no centro, sudeste e noroeste do estado. Para MAM notou-se que o modelo se mostrou representativo em grandes faixas da região sul e noroeste do estado. Para DJF a grade G2 do modelo mostra-se representativa para áreas no oeste, nordeste e noroeste do estado. Para MAM notou-se que o modelo mostrou-se representativo para a maior parte do estado com exceção da faixa que vai do nordeste ao noroeste do estado. Para DJF a grade G3 do modelo mostra-se representativa para parte da região noroeste e nordeste, e em algumas faixas do centro do Pará MAM notou-se que o modelo superestima os índices de chuva em grande parte do estado, com exceção de parte da região noroeste onde ele subestima os índices, mostrando-se representativo apenas em uma pequena faixa no noroeste do estado.

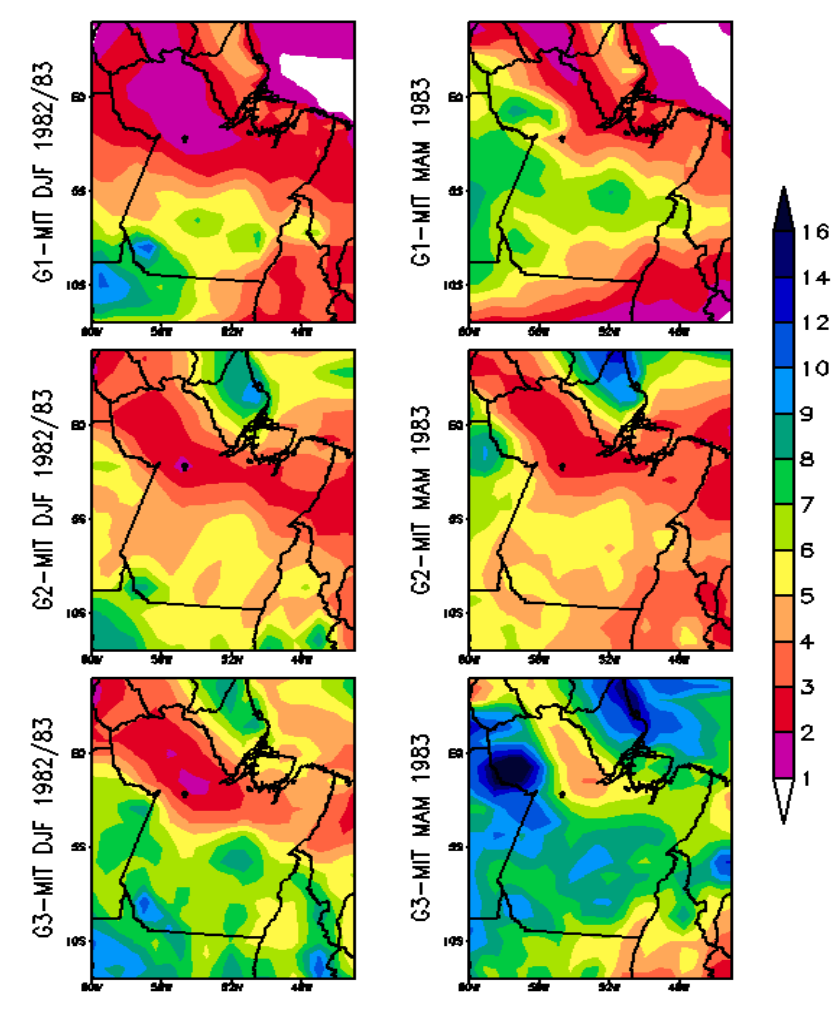

Figura 11. Padrões (distribuição espacial e intensidade) da precipitação ( $\mathrm{mm} / \mathrm{dia}$ ) sazonal simulada em DJF 1982/83 e MAM 1983 correspondentes às grades G1 (mapa superior), G2 (mapa intermediário) e G3 (mapa inferior), usando o esquema convectivo MIT. 
A Figura 14 mostra os padrões de precipitação simulados pelo modelo RegCM4 para DJF 1988/89 e MAM 1989 considerando as grades G1, G2 e G3, usando o esquema de parametrização convectiva MIT. Para DJF a grade G1 o modelo subestimou a intensidade da chuva em uma faixa que abrange parte do noroeste, norte e nordeste do estado, por outro lado o inverso pode ser verificado nas demais áreas do estado. Para DJF a grade G2 o modelo mostrouse representativo para parte da Ilha do Marajó em parte da região sudoeste no entanto ele subestima uma grande área na região noroeste do estado. Para MAM o modelo é representativo para uma boa parte da região oeste e centro do estado, enquanto que pra as demais áreas ele superestima os índices de chuva, com exceção de uma parte do noroeste em que ele subestima. No trimestre DJF a grade G3 o modelo mostra-se representativo para pequenas áreas no noroeste, ilha do Marajó e nordeste do estado, não sendo representativo para as regiões central e sul do estado devido a sua superestimativa da chuva. Para MAM o modelo mostra- se representativo para pequenas áreas na ilha do Marajó e nordeste do estado, não sendo representativo para as regiões oeste, central e sul do estado devido a sua superestimativa da chuva. Em uma analise pontual observa-se que as grades $\mathrm{G1}$ e G2 para DJF subestimam os valores pluviométricos para Belém e Santarém, no entanto para Marabá temos uma superestimativa na G1 e uma representatividade na grade G2. Para a grade G3 no trimestre de DJF o modelo superestima os índices de chuva para Santarém e Marabá e representa o observado para Belém e para MAM é representativo pra Belém, superestima para Marabá e subestima os índices de chuva para Santarém.

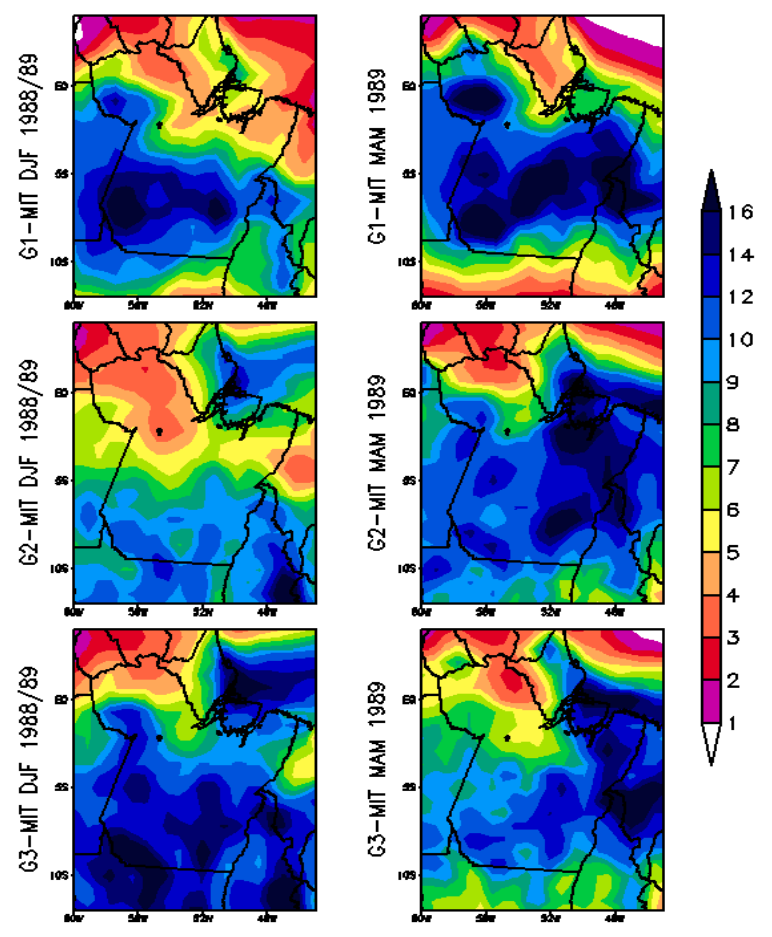

Figura 12. Padrões (distribuição espacial e intensidade) da precipitação ( $\mathrm{mm} / \mathrm{dia}$ ) sazonal simulada em DJF 1988/89 e MAM 1989 correspondentes as grades G1 (mapa superior), G2 (mapa intermediário) e G3 (mapa inferior), usando o esquema convectivo MIT. 


\section{CONCLUSÕES}

O presente trabalho abordou duas áreas igualmente importantes para aperfeiçoar os estudos em climatologia: a área observacional, a qual indiscutivelmente será sempre necessária para comprovar as teorias e ratificar as hipóteses da ciência; e a modelagem numérica, que tem se mostrado a cada dia uma das ferramentas mais poderosas no entendimento dos mecanismos físicos, particularmente, quando tratamos da atmosfera. A contribuição resultante desta pesquisa, dedicada à Amazônia Oriental, particularmente focada no Pará, onde a disponibilidade de dados meteorológicos em um passado não muito recente era sinônimo de lacuna, alcançou seguramente seus objetivos iniciais. Mais do que isso, permitiu que a ciência avançasse no campo da climatologia sobre uma das mais relevantes variáveis meteorológicas para a região tropical, a precipitação.

Podemos verificar que o RegCM4/MIT foi bem mais representativo quando estudado o ano de El Niño na G2 pra DJF mostrando que o noroeste e parte do centro do estado com boas áreas correspondendo ao observado e para MAM temos representatividade em boa parte do centro ao sul do estado e subestimando as demais áreas.

O RegCM4 demonstrou ser capaz de simular a variabilidade sazonal da precipitação para o Pará durante a estação chuvosa (verão e outono austral) para os anos de contraste climáticos caracterizados como extremos chuvoso e seco. Contudo, ajustes nas parametrizações mostram-se necessários para não se distanciar muito dos dados observados.

\section{REFERÊNCIAS}

ALVES, L. M. Simulações da variabilidade do clima presente sobre a América do Sul utilizando um modelo climático regional. 2007, 98 f. Dissertação (Mestrado em Meteorologia) - Instituto Nacional de Pesquisas Espaciais, INPE, São Paulo. 2007.

COHEN, J. C. P; DIAS, M. A. F. S.; NOBRE, C. A. Aspectos climatológicos das linhas de instabilidade na Amazônia. Climanálise - Boletim de Monitoramento e Análise Climática, v.4, n.11, p.34-40. 1989.

DICKINSON, R. E.; HENDERSON-SELLERS, A.; KENNEDY, P. J. Biosphereatmosphere transfer scheme (BATS) version 1E as coupled to the NCAR Community Climate Model. Boulder,Colorado. NCAR Technical Report, NCAR/TN-387, 72f. 1993.

EMANUEL, K. A. A scheme for representing cumulus convection in largescale models. Journal of the Atmospheric Sciences, v.48, n.21, p.23132335. 1991.

EMANUEL, K.A.; Zivkovic-Rothman, M. Development and evaluation of a convection scheme for use in climate models. Journal of the Atmospheric Sciences, v. 56, p. 1766-1782, 1999. 
FRITSCH, J. M.; CHAPPELL, C. F. Numerical prediction of convectively driven mesoscale pressure systems. Part I: Convective parameterization. Journal of Atmospheric Sciences, v.37, p.1722-1733. 1980.

GAN, M. A.; KOUSKY, V. E. Vórtices ciclônicos da alta troposfera no oceano Atlântico Sul. Revista Brasileira de Meteorologia, v.1, p.19-28. 1986.

GRELL, G. A. Prognostic evaluation of assumptions used by cumulus parameterizations. Monthly Weather Review, v.121, p.764-787. 1993.

HOLTSLAG, A.; DE BRUIJN, E.; PAN, H. L. A high resolution air mass transformation model for short-range weather forecasting. Monthly Weather Review, v.118, p.1561-1575. 1990.

LIEBMANN, B. et al. Submonthly convective variability over South America and the South Atlantic convergence zone. Journal of Climate, v.12, p.1877-1891. 1999.

KALNAY, E. et al. NCEP/NCAR 40-year Reanalysis project. Bulletin of the American Meteorological Society, v. 77, p. 437-471, 1996.

KIEHL, J. T. et al. Description of the NCAR Community Climate Model (CCM3). Boulder, Colorado. NCAR Technical Note, NCAR/TN-420+STR, 152p. 1996.

KODAMA, Y. Large-scale common features of subtropical precipitation zones (the Baiu frontal zone, the SPCZ, and the SACZ). Journal of the Meteorological Society of Japan, v.70, n.4, p.813-836. 1992.

MARENGO, J.A. et al. Onset and end of the rainy season in the Brazilian Amazon basin. Journal of Climate, v. 14, p. 833- 852, 2001.

NOBRE, P.; SHUKLA, J. Variations of SST, wind stress and rainfall over the tropical Atlantic and South America. Journal of Climate, v. 9, p. 2464-2479, 1996.

NOGUÉS-PAEGLE, J.; MO, K. C. Alternating wet and dry conditions over South America during summer. Monthly Weather Review, v.125, p.279-291. 1997.

REYNOLDS, R.W. et al.An improved in situ and satellite SST analysis for climate. Journal of Climate, v. 15, p. 1609-1625, 2002.

SETH, A. et al. RegCM3 regional climatologies for South America using reanalysis and ECHAM global model driving fields. Climate Dynamic, v.28, p.461-480. 2007.

SILVA, V. B. S. et al.An improved gridded historical daily precipitation analysis for Brazil. Journal of Hydrometeorology, v.8, p.847-861. 2007.

SOUZA, E.B. et al. On the influences of the El Niño, La Niña and Atlantic dipole pattern on the Amazonian rainfall during 1960-1998. Acta Amazonica, v. 30, n. 2, p. 305-318, 2000. 
SOUZA, E.B.; AMBRIZZI, T. ENSO impacts on the South American rainfall during 1980s: Hadley and Walker circulation. Atmósfera, v. 15, p. 105-120, 2002.

SOUZA, E. B.; AMBRIZZI, T. Pentad precipitation climatology over Brazil and the associated atmospheric mechanisms. Climanálise - Boletim de Monitoramento e Análise Climática, v.1. 2003.

SOUZA, E.B.; KAYANO, M.T.; AMBRIZZI, T. The regional precipitation over the eastern Amazon/northeast Brazil modulated by tropical Pacific and Atlantic SST anomalies on weekly timescale. Revista Brasileira de Meteorologia, v. 19, n. 2, p. 113-122, 2004.

SOUZA, E.B.; KAYANO, M.T.; AMBRIZZI, T. Intraseasonal and submonthly variability over the eastern Amazon and Northeast Brazil during the autumn rainy season. Theoretical and Applied Climatology, v. 81, p. 177-191, 2005.

SOUZA, E.B.; ROCHA, E.J.P. Diurnal variations of rainfall in Bragança-PA (eastern Amazon) during rainy season: mean characteristics and extreme events. Revista Brasileira de Meteorologia, v. 21, n. 3a, p. 142-152, 2006.

WALISER, D. E.; GAUTIER, C. A satellite-derived climatology of the ITCZ. Journal of Climate, v.6, n.11, p.2162-2174. 1993. 